\title{
CELL LOCATIONS FOR AQP1, AQP4 AND 9 IN THE NON-HUMAN PRIMATE BRAIN
}

\author{
I. I. ARCIÉNEGA, ${ }^{\text {a }}$ J. F. BRUNET, ${ }^{\text {a J. BLOCH }}$ AND \\ J. BADAUT ${ }^{\mathrm{a}, \mathrm{b}, \mathrm{c} *}$ \\ ${ }^{a}$ Neurosurgery Research Group, Lausanne Hospital University \\ (CHUV), Pavillon 3, 1011 Lausanne, Switzerland \\ ${ }^{b}$ Departments of Pediatrics, Physiology and Pharmacology, Loma \\ Linda University School of Medicine, Loma Linda, CA 92354, USA \\ 'Neurovascular Laboratory, Departments of Clinical and Fundamental \\ Neurosciences, Faculty of Medicine, Geneva University, Service of \\ Neurosurgery, 1211 Geneva, Switzerland
}

\begin{abstract}
The presence of three water channels (aquaporins, AQP), AQP1, AQP4 and AQP9 were observed in normal brain and several rodent models of brain pathologies. Little is known about AQP distribution in the primate brain and its knowledge will be useful for future testing of drugs aimed at preventing brain edema formation. We studied the expression and cellular distribution of AQP1, 4 and 9 in the nonhuman primate brain. The distribution of AQP4 in the nonhuman primate brain was observed in perivascular astrocytes, comparable to the observation made in the rodent brain. In contrast with rodent, primate AQP1 is expressed in the processes and perivascular endfeet of a subtype of astrocytes mainly located in the white matter and the glia limitans, possibly involved in water homeostasis. AQP1 was also observed in neurons innervating the pial blood vessels, suggesting a possible role in cerebral blood flow regulation. As described in rodent, AQP9 mRNA and protein were detected in astrocytes and in catecholaminergic neurons. However additional locations were observed for AQP9 in populations of neurons located in several cortical areas of primate brains. This report describes a detailed study of AQP1, 4 and 9 distributions in the non-human primate brain, which adds to the data already published in rodent brains. This relevant species differences have to be considered carefully to assess potential drugs acting on AQPs non-human primate models before entering human clinical trials. @ 2010 IBRO. Published by Elsevier Ltd. All rights reserved.
\end{abstract}

Key words: aquaporins, astrocyte, water homeostasis, brain edema, energy metabolism.

Aquaporins (AQPs) are a family of water channel proteins ubiquitously distributed in the living world (AmiryMoghaddam and Ottersen, 2003). To date, eight aquaporins are found to be expressed in the rodent brain: AQP1, AQP3, AQP4, AQP5, AQP6, AQP8, AQP9 and AQP11, but only AQP1, AQP4 and AQP9 have been clearly identified in brain cells in vivo (Badaut et al., 2007).

${ }^{*}$ Correspondence to: J. Badaut, Department of Pediatrics, Loma Linda University School of Medicine, Loma Linda, CA 92350, USA. Tel: +1-909-558-8242; fax: +1-909-558-0479.

E-mail address: jbadaut@llu.edu (J. Badaut).

Abbreviations: AQPs, aquaporins; GFAP, glial fibrillary acidic protein; $\mathrm{NMO}$, neuromyelitis optica; $\mathrm{SCl}$, spinal cord injury.
AQP4 was the first AQP reported in rat brain (Jung et al., 1994) and was shown to be highly expressed in perivascular astrocytic endfeet (Nielsen et al., 1997). Its presence in rodent brain was observed around all blood vessels of any size, in white and gray matter and in all brain structures (Badaut et al., 2000a). It is the dominant water channel localized in astrocyte foot processes, in contact with blood vessels and the cerebrospinal fluidbrain interface (pial and ependymal surfaces) (Badaut et al., 2002). Since 1997, AQP4 is the most studied AQP in mammalian brains and implicated in the physiopathology of brain edema, with a dual function depending on the pathological model used (Badaut et al., 2007; Tait et al., 2008). In mouse models of cytotoxic edema (water intoxication and stroke), AQP4 deletion has been shown to be protective by reducing edema formation and improving survival (Manley et al., 2000). It was therefore proposed to play a central role in the formation of cytotoxic brain edema. In vasogenic edema, AQP4 plays the opposite role where its deletion results in decreased edema clearance and further progression of disease (Papadopoulos et al., 2004). There is now more evidence that AQP4 is involved in human CNS pathologies. Indeed, recent reports demonstrate the presence of IgG recognizing AQP4 in the serum of patients suffering from neuromyelitis optica (NMO), an autoimmune demyelinating disease affecting optic nerves and the spinal cord (Lennon et al., 2005). This serum IgG autoantibody (IgG-NMO) served as a specific marker and was found to bind selectively to the extracellular face of the AQP4 water channel (Lennon et al., 2005). Furthermore, increased AQP4 expression was also observed in reactive astrocytes during glial scar formation and facilitated astrocyte migration (Saadoun et al., 2005b), as well as in other human pathologies such as astrocytomas and adenocarcinomas (Saadoun et al., 2002b); in sporadic Creutzfeldt-Jakob disease (sCJD) (Iwasaki et al., 2007; Rodriguez et al., 2006) and in HIV-related dementia (St Hillaire et al., 2005). On the other hand, decreased AQP4 expression was found in the cerebellum of subjects with autism (Fatemi et al., 2008a) and in animal models of autism, where prenatal influenza infection in mice resulted in a down regulation of AQP4 expression (Fatemi et al., 2008b, 2005).

AQP1 was first described in the choroid plexus of rodent and primate brains and thought to be involved in the production of cerebrospinal fluid (Oshio et al., 2005). AQP1 is also expressed throughout the body of mammalians in many endothelial cells (Nielsen et al., 1993), but its expression is suppressed in the specialized endothelial cells of the blood-brain barrier (Dolman et al., 2005).

0306-4522/10 \$ - see front matter @ 2010 IBRO. Published by Elsevier Ltd. All rights reserved.

doi:10.1016/j.neuroscience.2010.02.059 
Indeed, the presence of astrocytes inhibits the expression of AQP1 in endothelial cells (Dolman et al., 2005). Recently, the dorsal horn of the spinal cord and the trigeminal sensory ganglia were identified as new cellular locations for AQP1 (Nandasena et al., 2007; Oshio et al., 2006). In these areas, AQP1 may play a role in pain sensation as, interestingly, the expression of AQP1 in small diameter sensory fibers of the dorsal horn is locally significantly increased 10 days after spinal cord injury (SCI), persisting up to 11 months post-contusion. AQP1 is proposed to play two distinctive roles in the reaction after $\mathrm{SCl}$ : (i) at early time points post-SCl increase in expression contributes to astrocytic reactivity and neuronal swelling and (ii) the delayed increase in expression contributes to development of chronic neuropathic pain after SCI (Nesic et al., 2008).

In human, AQP1 expression was also found to be enhanced in reactive astrocytes with highly branched processes surrounding blood vessels, along the glial scar and accumulating in brain lesions in Alzheimer's disease, Creutzfeldt-Jakob disease, multiple sclerosis and in ischemic lesions (Misawa et al., 2008; Satoh et al., 2007). Under these pathological conditions, AQP1 is suggested to play a complementary role to AQP4 in brain water homeostasis (Misawa et al., 2008; Satoh et al., 2007). To date, regional and cellular distribution of AQP1 in healthy human and non-human primate brain has never been investigated in detail.

The aquaglyceroporin, AQP9 has been reported to be present in glial cells (Badaut et al., 2001; Elkjaer et al., 2000) and in catecholaminergic neurons (Badaut et al., 2004) in rodent brains. With regards to AQP9 localization and its permeability properties, AQP9 was hypothesized to play a role in brain energy homeostasis (Badaut and Regli, 2004). Interestingly, the level of AQP9 expression is increased in astrocytes after stroke (de Castro Ribeiro et al., 2006) and in catecholaminergic neurons in a diabetes model in rats (Badaut et al., 2008). Despite many publications which describe the presence of AQP9 in the rodent brain (Amiry-Moghaddam et al., 2005; Badaut et al., 2001, 2004; de Castro Ribeiro et al., 2006; Elkjaer et al., 2000; Tsukaguchi et al., 1998), it has now became a matter of debate following a recent publication (Rojek et al., 2007), suggesting that AQP9 is not expressed in the mouse brain and raising the question of its expression in primate brain.

Most investigations have been carried out in rodent brains (mice and rats) and there is no description of AQPS in normal primate brains. Cells in the CNS differ between rodents and primates, for example: human protoplasmic astrocytes manifest a threefold larger diameter and have tenfold more primary processes than those of rodents (Oberheim et al., 2006). Since AQP1, 4 and 9 are all found in astrocytes (Badaut et al., 2007), it is important to investigate possible evolutionary differences between rodents and primates, in the expression and distribution of AQPs. This knowledge will be essential for the development of future therapeutic agents targeting AQPs to prevent brain edema formation (Tait et al., 2008). Using RT-PCR and immunohistochemical approaches, we provide new data on the expression and regional, cellular distribution of AQP1, AQP4 and AQP9 in the primate brain.

\section{EXPERIMENTAL PROCEDURES}

\section{Tissue preparation}

The experiments were conducted on sections from three adult monkey brains (Macaca fascicularis). The animals were between 4 and 8.5 years old and weight $2.9-3.5 \mathrm{~kg}$. They lived in group of four in a space of $16 \mathrm{~m}^{3}$. They were fed once a day and had free access to water. Animal care was conducted in accordance with the NIH Guide for the care and use of laboratory animals (ISBN $0-309-05377-3 ; 1996)$ and was approved by the local Swiss veterinary authority.

The animals were killed under deep (lethal) anaesthesia (90 $\mathrm{mg}$ sodium pentobarbital/kg body weight, Sigma-Aldrich, St. Louis, MO, USA) by transcardiac perfusion with $0.9 \%$ saline $(400$ $\mathrm{ml}$ ). The perfusion was continued with fixative (3। of $4 \%$ phosphate-buffered paraformaldehyde (Sigma-Aldrich, St. Louis, MO, USA) in $0.1 \mathrm{M}$ phosphate buffer, $\mathrm{pH} 7.6$ ) and solutions (2 I each) of the same fixative containing increasing concentrations of sucrose $(10 \%, 20 \%$, and $30 \%)$. The brain was dissected and placed in a $30 \%$ sucrose in phosphate buffer solution for cryoprotection for 7 days. Fifty micrometres thick sections were prepared with a cryotome (HM560, MICROM, Volketswil, Switzerland) and were stored at $-80{ }^{\circ} \mathrm{C}$ in cryoprotection solution $(50 \mathrm{mM}$ phosphate buffer $\mathrm{pH} 7.4$ ) containing $25 \%$ glycerol (G7893, Sigma-Aldrich, St Louis, MO, USA) and 30\% ethylene glycol (33068, Ridel-de-Haën, Seelze, Germany).

\section{Tissue analysis}

The Macaca fascicularis brains were analyzed by steps of $1 \mathrm{~mm}$ coronal sections, beginning at $-32 \mathrm{~mm}$ and pursuing until $+7 \mathrm{~mm}$ based on the Atlas of the primate brain published from the Washington University (http://braininfo.rprc.washington.edu/). Most attention was given to coronal sections between -5 and $-13 \mathrm{~mm}$. These sections contain cerebral structures that have previously been studied in rodents and where thus useful to serve as comparison in our studies. Cerebellum was not included in our studies.

\section{Real Time Nested-PCR experiments}

mRNA was extracted from fixed brain slices from the three Macaca fascicularis monkeys adjacent to slices of interest used for immunohistochemistry using RNeasy ${ }^{\circledR}$ FFPE extraction kit (Qiagen, Hilden, Germany) and following the procedure proposed by the company. The quality of the mRNA was assessed using a bioanalyzer 2100 (Agilent Technologies, Santa Clara, CA, USA). cDNAs were generated from $500 \mathrm{ng}$ of total mRNA using Biolabs M-MuLV Reverse Transcriptase $(200 \mathrm{U} / \mu \mathrm{l})$ as described by the supplier. PCR experiments were performed using the HotStarTaq Master Mix Kit from Qiagen using $2 \mu \mathrm{l}$ of cDNA/5 pmol of primer and 35 cycles using a Thermocycler (Biometra, Goettingen Germany) with denaturation $\left(94{ }^{\circ} \mathrm{C}, 30 \mathrm{~s}\right)$, annealing $\left(48{ }^{\circ} \mathrm{C}, 45 \mathrm{~s}\right.$ for AQP 1,4 and 9 and $56^{\circ} \mathrm{C}, 45 \mathrm{~s}$ for Actin) and extension $\left(72{ }^{\circ} \mathrm{C}\right.$, $80 \mathrm{~s}$ ). The Nested-PCR reaction (not used for Actin amplification) used $1 \mu \mathrm{l}$ of the first PCR product with $5 \mathrm{pmol}$ of primer and consisted of 30 cycles in the same conditions as for the first PCR reactions. Primers used were specific for human AQP1, human AQP4, human AQP9 and human Actin and are specified in Table 1. Specificity of PCR products were controlled by sequence analysis (data not shown)

\section{Single immunolabeling for regional studies}

Immunolabeling was carried out in PBS containing $0.3 \%$ Triton $\mathrm{X}-100$ and $0.25 \%$ bovine serum albumin on adjacent serial 50 - 
Table 1. Gene-specific N-PCR primers and their respective fragment size

\begin{tabular}{|c|c|c|c|c|}
\hline GenBank accession & Primer & Nucleotide sequence & Position & Final fragment size \\
\hline \multirow[t]{4}{*}{ U41517 } & Human AQP1 Fo1 & 5'-GTC CAG GAC AAC GTG AAG GT-3' & 169-189 & $372 \mathrm{bp}$ \\
\hline & Human AQP1 Re1 & 5'-CCC TCC ACT GCA CCT CCT GG-3' & $1306-1325$ & \\
\hline & Human AQP1 Fo2 & 5'-ACC GCG TGA AGG TGT GGA CC-3' & $752-772$ & \\
\hline & Human AQP1 Re2 & 5'-ACT AGC AGG TGG GTC CCT TT-3' & $1104-1124$ & \\
\hline \multirow[t]{4}{*}{ D63412 } & Human AQP4 Fo1 & 5'-TTG CTT TGG ACT CAG CAT TG-3' & $245-264$ & $298 \mathrm{bp}$ \\
\hline & Human AQP4 Re1 & 5'-CTG TTG TCC TCC ACC TCC AT-3' & $852-871$ & \\
\hline & Human AQP4 Fo2 & 5'-CTG TCT TCT ACA TCG CAG CCC AG-3' & $364-386$ & \\
\hline & Human AQP4 Re2 & 5'-GGG ATT CAT GCT GGC ACC AG-3' & $643-662$ & \\
\hline \multirow[t]{4}{*}{ AB008775 } & Human AQP9 Fo1 & 5'-GCC CAA GCT ATT CTC AGT CG-3' & 349-369 & $364 \mathrm{bp}$ \\
\hline & Human AQP9 Re1 & 5'-GCA GAC AAA ACT GGG TGG AT-3' & $1197-1216$ & \\
\hline & Human AQP9 Fo2 & 5'-GTC CTT TGC TGG TGG AAA ACT GC-3' & $603-625$ & \\
\hline & Human AQP9 Re2 & $5^{\prime}-$ GGC CCA CTA CAG GAA TCC ACC-3' & $947-967$ & \\
\hline
\end{tabular}

Fo, forward; Re, reverse.

$\mu \mathrm{m}$-thick coronal free floating sections of Macaca fascicularis brain. After each incubation, sections were rinsed in PBS $3 \times 10$ $\min$. The sections were first incubated overnight at $4{ }^{\circ} \mathrm{C}$ with rotation with anti-AQP1 (dilution: 1/200; Chemicon, Temecula, CA, USA), anti-AQP4 (dilution: 1/200; Chemicon, Temecula, CA, USA) and anti-AQP9 (dilution: 1/200; Alpha Diagnostics, San Antonio, TX, USA). After washing, floating sections were then incubated for $2 \mathrm{~h}$ at room temperature with a biotinylated secondary goat anti-rabbit antibody (dilution: 1/200; Vector Laboratories, Burlingame, CA, USA). The sections were then rinsed and exposed for $1 \mathrm{~h}$ to avidin-biotin complex solution (Vectastain kit; Vector Laboratories, Burlingame, CA, USA) before application of diaminobenzidine (DAB; Vector kit, Vector Laboratories). Floating sections were rinsed, mounted on slides and then coverslipped with Eukit Medium. Controls were performed by omitting primary antibody. Control sections showed no detectable labeling.

\section{Double immunofluorescence}

Sections were incubated with either one primary antibody (see Table 2) or a mixture of two primary antibodies over night at $4{ }^{\circ} \mathrm{C}$ with rotation and then with either one secondary antibody or a mixture of two secondary antibodies: anti-rabbit coupled to Alexadye $594 \mathrm{~nm}$ (dilution: 1/1000, Invitrogen, Switzerland) and antimouse coupled to Alexa-dye $488 \mathrm{~nm}$ (dilution: 1/1000, Invitrogen, Switzerland) for $2 \mathrm{~h}$ at room temperature. After immunofluorescence, sections were mounted and coverslipped with mounting medium Vectashield (Vector Laboratories, Burlingame, CA, USA). Controls were performed by omitting either one or both primary antibodies. Control sections showed no detectable labeling.

Table 2. Antibodies and dilutions used in this study

\begin{tabular}{lc}
\hline Primary antisera & Secondary antisera \\
\hline $\begin{array}{c}\text { Anti-AQP1 (Chemicon, rabbit, } \\
1 / 200)\end{array}$ & $\begin{array}{c}\text { Alexa } 594 \text { goat anti-rabbit } \\
\text { (Invitrogen, 1/1000) }\end{array}$ \\
$\begin{array}{c}\text { Anti-AQP4 (Chemicon, rabbit, } \\
\text { 1/200) }\end{array}$ & $\begin{array}{c}\text { Alexa } 488 \text { goat anti-mouse } \\
\text { (Invitrogen, 1/1000) }\end{array}$ \\
$\begin{array}{c}\text { Anti-AQP9 (Alpha Diagnostics, } \\
\text { rabbit, 1/200) }\end{array}$ & $\begin{array}{c}\text { CY3 goat anti-rabbit (Sigma, } \\
1 / 1000)\end{array}$ \\
Anti-GFAP (Sigma, mouse, 1/500) & $\begin{array}{c}\text { Alexa 488 goat anti-mouse } \\
\text { (Invitrogen, 1/1000) }\end{array}$ \\
Anti-TH (Chemicon, mouse, 1/20) & FITC goat anti-mouse (Sigma, \\
& $1 / 1000)$ \\
Anti-NF 70+200 kDa (Bio-Science & Alexa 488 goat anti-mouse \\
Products, mouse, 1/100) & (Invitrogen, 1/1000) \\
\hline
\end{tabular}

\section{Image acquisitions}

The entire section of a single immunostaining was scanned by a HP Scanjet 4850 with a resolution of 600 pixels/inch to obtain a single picture of the whole section.

Single immunolabeling revealed by DAB staining was observed and photographed using optical microscopy (Olympus BX40, Switzerland). Immunofluorescent preparations were examined using epifluorescence microscopy (Olympus BX 40, Switzerland), equipped with a Qimagin, QICAM Fast (1394) camera and ExploraNova Fluo 3D software (ExploraNova, La Rochelle, France). The epifluorescence of FITC, CY3, Alexa 488 and Alexa 594 was successively photographed through different filters and then superposed.

RT-PCR images were acquired with an infrared Scanner (Odyssey, from Licor, Germany) detecting Syto60 and using software Odyssey 1.2 .

\section{RESULTS}

\section{Detection of AQP4, 1 and 9 mRNAs in Macaca fascicularis brain}

The expression of AQP4, 1 and 9 mRNAs was first evaluated by RT-PCR using fixed Macaca fascicularis brain slices, adjacent to the slices used for immunohistochemical studies. Fragments of the expected size were obtained for AQP4, 1 and 9 cDNAs respectively 298, 372 and 364 bp from all the three Macaca fascicularis brains (Fig. 1). Large amounts of AQP1 and 4 cDNA were detected,

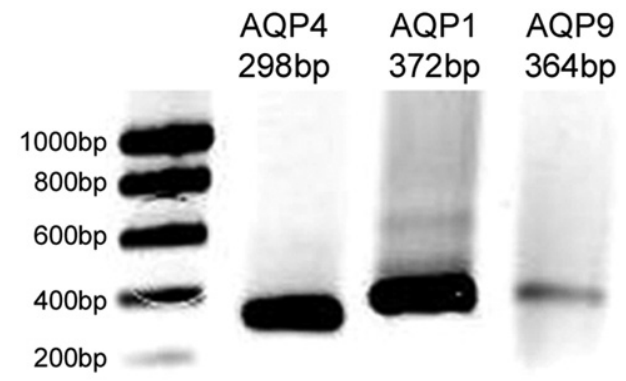

Fig. 1. Real time PCR analysis of fixed Macaca fascicularis brain tissue. Fragments of expected sizes for AQP4, 1 and 9 respectively 298, 372 and 364 bp were obtained. A large amount of AQP1 and 4 mRNA is detected, whereas we observe a weak, but specific signal for AQP9 mRNA. 

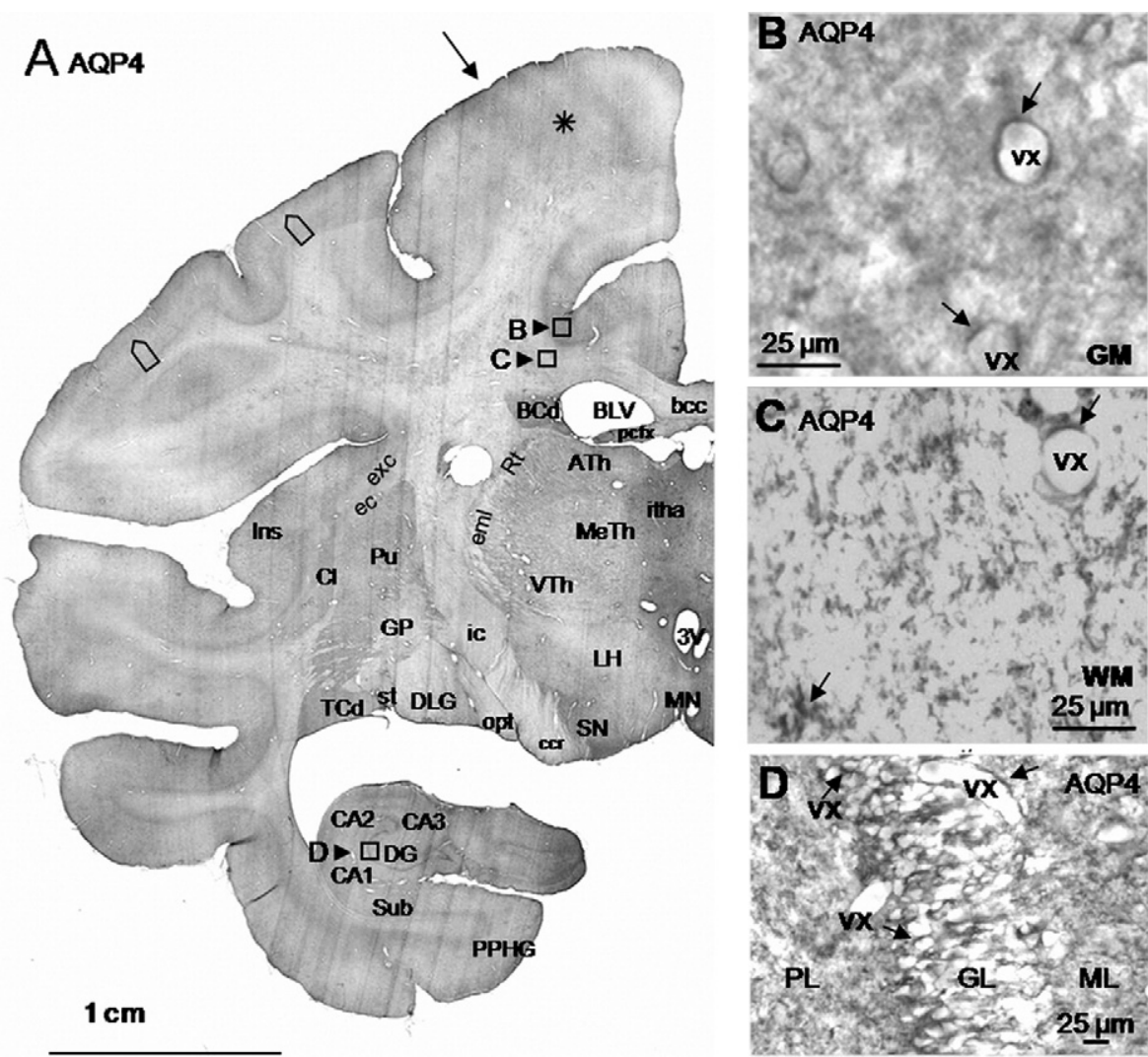

Fig. 2. AQP4-immunoreactivity in a Macaca fascicularis coronal section at anterior commissure (AC) $-5 \mathrm{~mm}$. (A) Immunolabeling of AQP4 is observed in grey matter (asterix), in the anterior thalamic nuclear group (ATh), in the ventral (VTh) and medial (MeTh) thalamic nuclear group, in the substantia nigra ( $\mathrm{SN}$ ), in the putamen ( $\mathrm{Pu}$ ), in the interthalamic adhesion (itha), in the globus pallidus (GP), in the tail (TCd) and body (BCd) of the caudate nucleus, in the granule cell layer of the dentate gyrus (DG), in the glia limitans, in the pia mater (single lined arrow) and in the posterior column of fornix (pcfx). 3V, third ventricle; Ath, anterior thalamic nuclear group; BLV, body of lateral ventricle; CA1, CA2 and CA3, Cornu Ammonis fields; ccr, cerebral crus; $\mathrm{Cl}$, claustrum; DLG, dorsal nucleus of lateral geniculate body; ec, external capsule; exc, extreme capsule; ic, internal capsule; Ins, Insula; itha, interthalamic adhesion; LH, lateral hypothalamic area; MN, mammillary nuclei; opt, optic tract; PPHG, posterior parahippocampal gyrus; Rt, reticular thalamic nuclei; st, stria terminalis; Sub, subiculum; eml, external medullary lamina. Higher magnification of several zones of Fig. $2 \mathrm{~A}$ (indicated by open boxes A, B, C) and comparison of AQP4 immunolabeling in grey (GM) and white matter (WM). (B) In grey matter, AQP4 staining polarizes to the astrocytic perivascular endfeet (arrows). (C) In white matter, AQP4 staining also polarizes to the astrocytic perivascular endfeet (arrows). Fewer vessels are present in white matter compared to grey matter. (D) Higher magnification of the indicated zone in Fig. 2A shows AQP4-immunoreactivity (IR) in the granule cell layer $(\mathrm{GL})$ of the dentate gyrus in the hippocampus. AQP4 staining in the granule cell layer labels also perivascular astrocytic endfeet (arrows). $\mathrm{PL}$, polymorphic layer; $\mathrm{GL}$, granule cell layer; $\mathrm{ML}$, molecular cell layer; $\mathrm{vx}$, vessels. $\mathrm{Bar}=1 \mathrm{~cm}$ in $(\mathrm{A})$; bar=25 $\mu \mathrm{m}$ in $(\mathrm{B}, \mathrm{C}, \mathrm{D})$.

whereas a weak, but specific signal was obtained for AQP9 cDNA (Fig. 1) demonstrating the presence of AQP9 mRNA in Macaca fascicularis brain on adjacent slices used for immunolabeling (see below).

\section{AQP4 immunolabeling in astrocytic perivascular endfeet}

AQP4 labeling was systematically studied in all structures of the fixed Macaca fascicularis brain. Low magnification (Fig. 2A) showed that AQP4 staining was present in grey matter (GM), in several brain areas such as the anterior thalamic nuclear group (ATh), the ventral (VTh) and medial (MeTh) thalamic nuclear group, the substantia nigra (SN), the putamen $(\mathrm{Pu})$, the globus pallidus (GP), the tail (TCd) and body $(B C d)$ of the caudate nucleus and the granule cell layer of the dentate gyrus (DG). High AQP4 staining was also observed in the glia limitans, pia mater and the posterior column of fornix (pcfx). The wide distribution of
AQP4 staining is in accordance with the intense band observed in RT-PCR experiments (Fig. 1).

Higher magnification of grey and white matter (Fig. 2B, C) revealed, as in rodents, that AQP4 staining is polarized in the astrocytic perivascular endfeet along blood vessels. A higher vascular density in the grey matter compared to the white matter might explain why AQP4-immunoreactivity (AQP4-IR) was stronger in the grey matter (Fig. 2A). In the dentate gyrus of the hippocampus, AQP4 staining was observed in astrocyte processes in contact with blood vessels and also in astrocyte processes localized in between neuronal cell bodies (Fig. 2D). AQP4 expression and distribution are comparable between rodent and primate brains, suggesting a conserved functional role in evolution.

\section{AQP1 distribution in Macaca fascicularis brain}

AQP1 labeling was systematically studied in all structures of fixed Macaca fascicularis brain. In accordance with pre- 


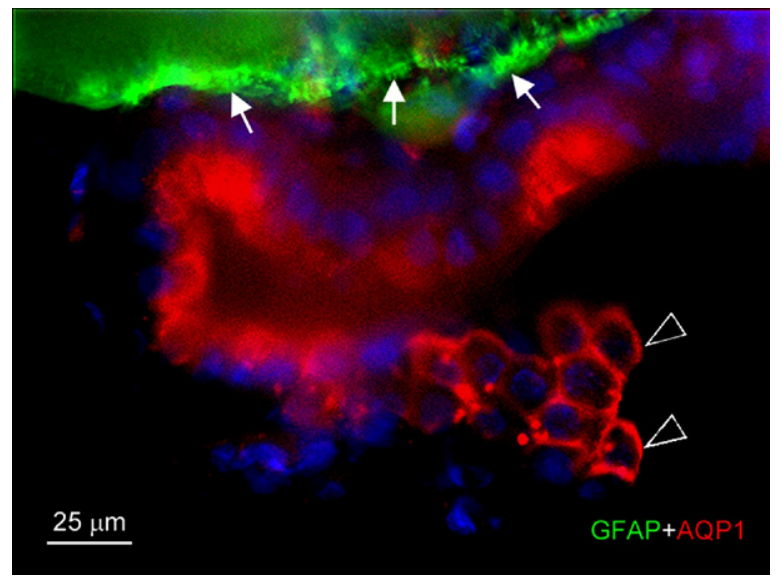

Fig. 3. Double immunohistochemistry with anti-GFAP (green, arrows), anti-AQP1 (red, arrowheads) antibodies and DAPI nucleus staining (blue). This experiment reveals the specificity of the antiAQP1 antibody for epithelial cells of choroid plexus in accordance with previous publications. Bar $=25 \mu \mathrm{m}$. For interpretation of the references to color in this figure legend, the reader is referred to the Web version of this article.

vious studies performed in rodent brains (de Castro Ribeiro et al., 2006; Gomori et al., 2006), immunolabeling with anti-AQP1 antibodies was observed in the choroid plexus epithelial cells (Fig. 3), showing that the commercial APQ1 antibody specifically recognized the AQP1 epitope in primate brains.

AQP1 immunolabeling (Fig. 4A) was not only restricted to the choroid plexus, but was also detected in white matter tracts, including the subcortical white matter, the internal (ic), external (ec) and extreme (exc) capsule and in the cerebral crus (ccr) (Fig. 4A). AQP1 staining was also observed around the optic tract (opt), in the tail (TCd) and body (BCd) of the caudate nucleus, in the lateral medullary lamina which separates the putamen $(\mathrm{Pu})$ from the globus pallidus (GP), in the hippocampus Cornu Ammonis fields CA1 to CA3 and in the granule cell layer of DG (Fig. 4A). It is interesting to note that AQP1 immunoreactivity does not overlap with AQP4 staining (Figs. 2A and 4A) in several regions (white and grey matter, medial (MeTh) and ventral (VTh) thalamic nuclear group, substantia nigra $(\mathrm{SN})$, putamen $(\mathrm{Pu})$, cerebral crus (ccr)).

Observations at higher magnification of the outlined regions shown in Fig. 4A revealed that AQP1-IR in grey and white matter was both due to an astrocyte-like labeling, with staining on cellular processes and perivascular endfeet (Fig. 4B, C). Higher magnification of the hippocampus (Fig. 4D) revealed a strong AQP1-immunolabeled granule cell layer of the DG with immunolabeled cell bodies and processes reaching from the granule cell layer into the molecular layer of the DG.

Double immunofluorescence with anti-glial fibrillary acidic protein (GFAP) and anti-AQP1 antibodies showed co-localization of these markers in the perivascular astrocytes suggesting that AQP1 is expressed in astrocytes (Fig. 5A-C). Astrocytic AQP1 immunoreactivity could also be seen in the glia limitans (Fig. 5A-C). In the white matter (Fig. 4C), we also note immunoreactivity in stellate cell bodies, suggesting expression in astrocytes. These labeled astrocytic soma contribute to the strong labeling in white matter (Fig. 4A, C). Comparison of GFAP and AQP1 staining in Fig. $5 \mathrm{~A}$ shows that not all GFAP positive cells were labeled AQP1. This result is in contrast to AQP4-IR which is observed in all GFAP stained astrocytes (data not shown). Here, we provide evidence that AQP1 may be a marker of a distinctive subpopulation of astrocytes present in the white matter of the primate brain.

Higher magnification of the emerging oculomotor nerve (Fig. 5D, E) revealed GFAP-AQP1-IR between the nerve fibers; a space filled by Schwann cells and endoneurium. Both nerve fibers and Schwann cells are enveloped by endoneurium, a loose connective tissue rich in vasa nervorum. Previous studies showed GFAP-labeling around nerve fibers in Schwann cells (Jessen and Mirsky, 1984; Jessen et al., 1990). Thus, AQP1 and GFAP co-localization in Schwann cells (Fig. 5D, E) could indicate a role in axon to Schwann cell signaling (Jessen et al., 1990), important for the maintenance of the nerve fiber microenvironment and its physiological functioning.

Interestingly, AQP1 staining without GFAP labeling was present on pial blood vessels (Fig. 5D, F). We noted AQP1-IR depicting soma as well as prolongations (Fig. $5 F$ ). Double immunolabeling with anti-Neurofilament (NF) antibodies clearly showed that AQP1 labeling is located in neuronal soma (Fig. 6) and their bipolar processes running around the blood vessels (Figs. 5F and 6). AQP1 labeling in these processes surrounded the NF staining, suggesting that AQP1 was inserted in the cell membrane of these neurons which innervate the pial blood vessels (Fig. 6).

\section{AQP9 immunolabeling in catecholaminergic neurons, in cortical isolated neurons and in astrocytes}

AQP9 immunolabeling was systematically studied in all structures of fixed Macaca fascicularis brain. Regional observations (Fig. 7A) revealed AQP9 immunoreactivity predominantly in the grey matter, the medial (MeTh) and ventral (VTh) thalamic nuclear group, the lateral geniculate body (DLG), the substantia nigra (SN), the tail (TCd) and body $(\mathrm{BCd})$ of the caudate nucleus, the Cornu Ammonis fields CA1 to CA3, the granule cell layer of the DG and the subiculum (Sub) of the hippocampus.

In the insula cortex (Fig. 7B), the precentral gyrus (Fig. 7C) and other brain areas, AQP9 staining was located in the astrocytic cell bodies and processes running towards vessels and in their perivascular endfeet. This AQP9 localization is in agreement with previous publications in rodent brains (Badaut et al., 2001, 2004), and double immunolabeling with GFAP shows co-localization of both markers (data not shown).

AQP9 immunoreactivity was also observed in several neurons (Figs. 7D and 8), indicating TH-positive neurons located in the substantia nigra (Fig. 8), in agreement with observations in rodent brains (Badaut et al., 2004; de Castro Ribeiro et al., 2006) where AQP9 expression was seen in catecholaminergic neurons. In these neurons, AQP9 labeling was observed in the cell membrane as well as in the cytoplasm (Fig. 8). In primates, additional AQP9 

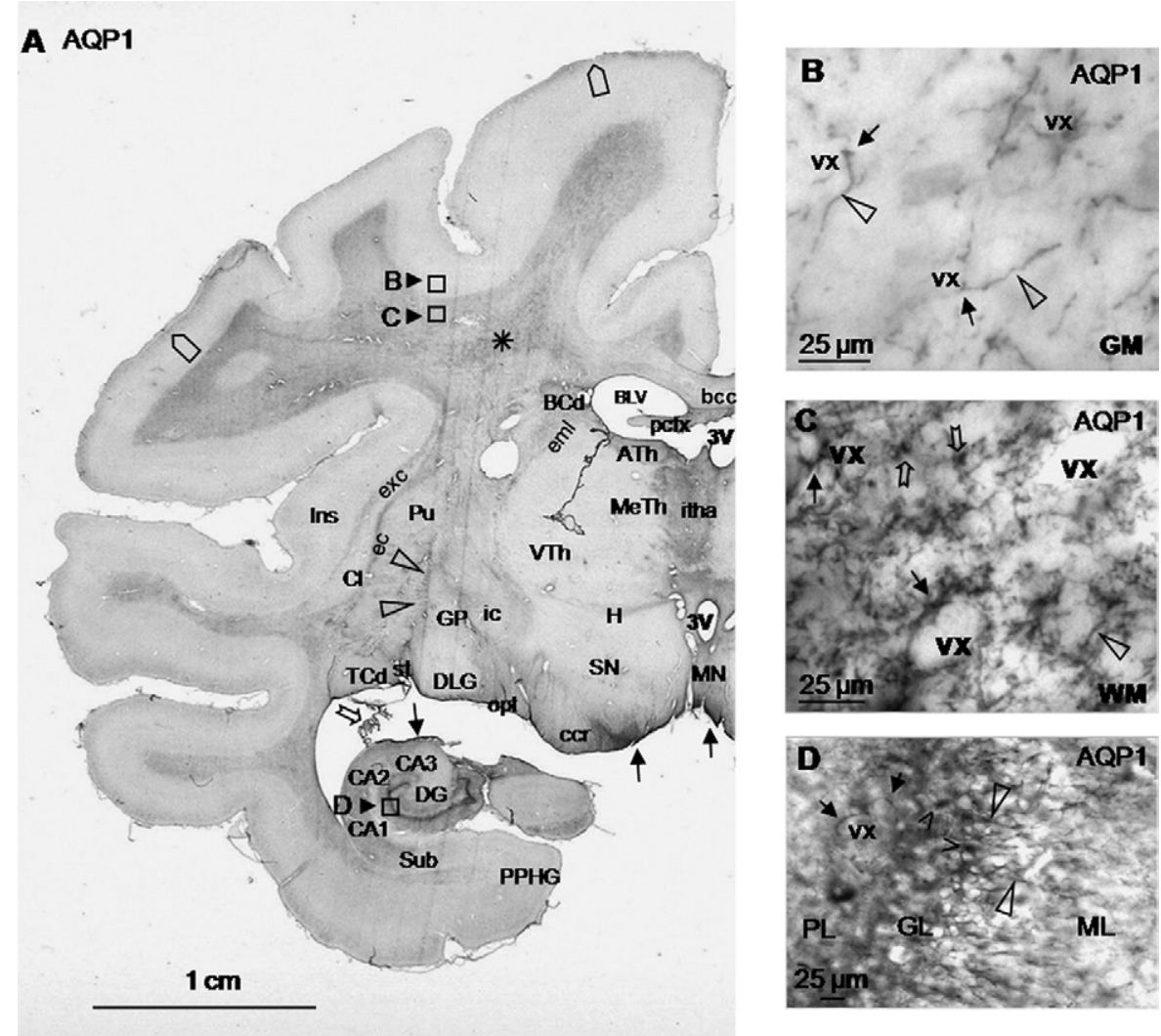

Fig. 4. AQP1-immunoreactivity in a Macaca fascicularis coronal section at $-5 \mathrm{~mm}$. (A) Immunolabeling is found in the choroid plexus (double lined arrow), in white matter (asterix) such as in the extreme (exc), external (ec) and internal capsule (ic), in the cerebral crus (ccr) which is the anterior part of the cerebral peduncle containing corticospinal, corticopontine and corticobulbar motor tracts, around the optic tract (opt) and the stria terminalis (st.), as well as in the lateral medullary lamina (arrow heads), separating the putamen (Pu) from the globus pallidus (GP) and in the external medullary lamina (eml), separating the ventral thalamic nuclear group (VTh) from the thalamic reticular nucleus (Rt). Other structures like the glia limitans (pentagonal arrows), and pia mater (single lined arrows) surrounding the glia limitans, are labeled. Immunoreactivity is also revealed around the tail (TCd) and body (BCd) of the caudate nucleus, in the interthalamic adhesion (itha), in the field $\mathrm{H}$, separating the ventral thalamic nuclear group (VTh) from the substantia nigra (SN), in Cornu Ammonis fields CA1, CA2 and CA3 and in the granular and molecular cell layers of the dentate gyrus (DG) of the hippocampus. Immunoreactivity is also strong in the posterior column of fornix (pcfx). 3V, third ventricle; ATh, anterior thalamic nuclear group; BLV, body of lateral ventricle; Cl, claustrum; DLG, dorsal nucleus of lateral geniculate body; Ins, Insula; MeTh, medial thalamic nuclear group; MN, mammillary nuclei; PPHG, posterior parahippocampal gyrus; Sub, subiculum. (B) A higher magnification of the grey matter (GM) reveals a few vessels ( $v x$ ) surrounded by immunolabeled perivascular endfeet (arrows) as well as their immunolabeled prolongations (arrow heads), suggesting the presence of astrocytes. (C) As seen in the coronal section, AQP1 staining is stronger in white matter (WM) than in grey matter (GM). In contrast to grey matter (GM), in white matter (WM) we also observe immunoreactivity in stellate cell bodies (double lined arrows), suggesting AQP1 expression in astrocytic cell bodies. These cell bodies show immunolabeled perivascular endfeet (single lined arrows) and immunolabeled prolongations (arrow heads). (D) AQP1-immunoreactivity in the hippocampus of Macaca fascicularis brain. The hippocampus region reveals a strongly immunolabeled granule cell layer (GL). Higher magnification allows identification of immunolabeled cell bodies (open arrow heads) in the granule cell layer of the dentate gyrus and immunolabeled processes (closed arrow heads) reaching from granule cell layer to the molecular layer (ML) of the DG. Single lined arrows show the staining in perivascular end feet, suggesting the presence of astrocytes. PL, polymorphic layer; GL, granule cell layer; ML, molecular cell layer. $\operatorname{Bar}=1 \mathrm{~cm}$ in $(\mathrm{A})$; $\operatorname{bar}=25 \mu \mathrm{m}$ in $(B-D)$.

positive neurons were also observed in the insula cortex as well as in other brain areas such as the fourth layer of the precentral gyrus (primary motor cortex) (Fig. 7D). AQP9labeled neurons exhibiting an oval shape were found located in the cortical layer IV (Fig. 7D). Interestingly, AQP9-IR was observed in the DG (Fig. 7A, E, F). Higher magnification revealed a strongly immunolabeled granule cell layer of the DG, with immunolabeled cell bodies and processes reaching from the granule cell layer into the molecular layer (Fig. 7E, F). Cell bodies were stained with AQP9 in the granular layer as well as in the polymorphic layer (Fig. 7F).

\section{DISCUSSION}

Interest in AQP expression in human pathologies is oriented towards the development of new drugs to prevent edema formation. Recently, the relevance to study AQP4 in human diseases has been strengthened by the discovery of the AQP4 antibody in blood of patient suffering of neuromyelitis optica (NMO), and is now a biomarker of this neurodegenerative disease (Lennon et al., 2005; Weinshenker et al., 2006). However, most AQP studies have been carried out in the rodent brain (Badaut et al., 2007, 2002; Tait et al., 2008; Verkman et al., 2006), but a small 


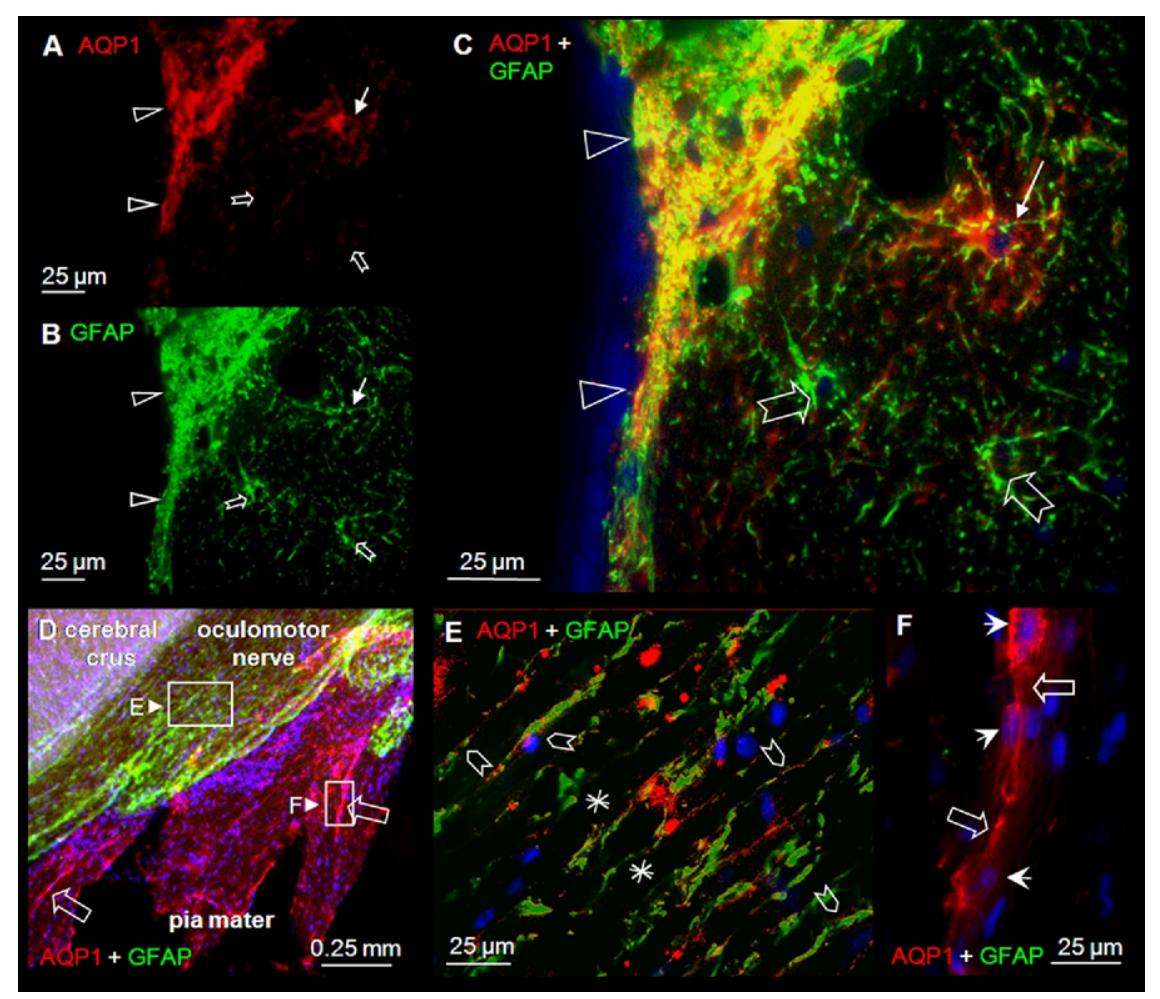

Fig. 5. (A-C) AQP1 (red)-GFAP (green) double immunofluorescence in the glia limitans. Large arrow heads indicate the co-localization (yellow) in the glia limitans. Single lined arrows point out an astrocyte with its double immunolabeled prolongations. We also observe astrocytic prolongations that are GFAP positive, but AQP1 negative (double lined arrows in Fig. 5A-C). (D) AQP1 staining in red, GFAP labeling in green and DAPI in blue. Double immunolabeling reveals AQP1 and GFAP immunoreactivities in the cerebral crus, a strongly GFAP stained oculomotor nerve and a predominant AQP1 immunoreactive pia mater, containing vascular structures (double lined arrows). (E) Higher magnification, of the zone indicated by an open box in Fig. 5D, allows the identification of GFAP (green)-AQP1 (red) positive Schwann cells (hexagonal-arrows) in the endoneurial space around the nerve fibers of oculomotor nerve axons (asterixes). (F) A closer look into the pia mater surrounding the emerging oculomotor nerve, reveals AQP1 immunolabeling of fibers (double lined arrows) and cell bodies (arrows) interconnected by the stained fibers, suggesting a neuronal formation which runs along a blood vessel. There is no detectable GFAP staining in the pia mater. Bar $=25 \mu \mathrm{m}$ in $(B-D, F)$; bar $=0.25 \mathrm{~mm}$. For interpretation of the references to color in this figure legend, the reader is referred to the Web version of this article.

number of reports have been published using human brain tissue samples, showing regulation of AQP ex-

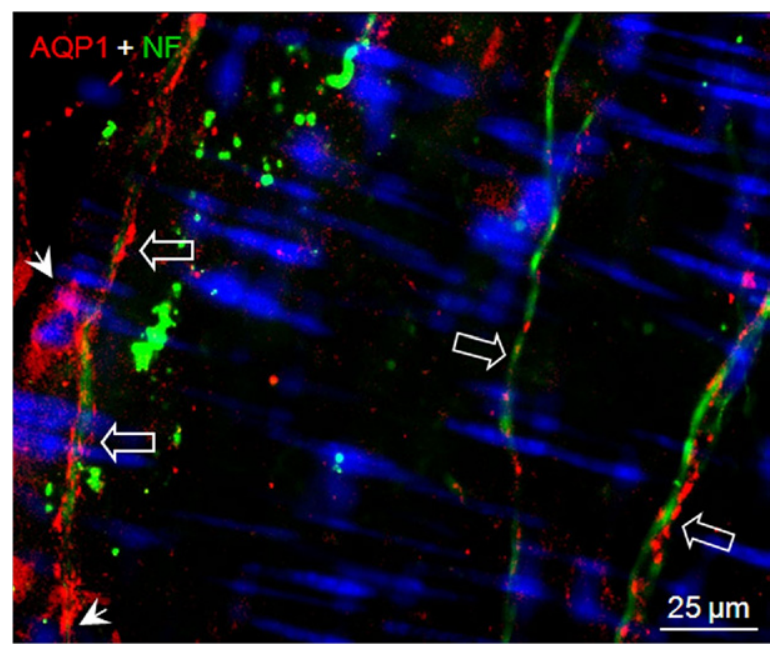

Fig. 6. AQP1-labeling (red) and neurofilament (NF, green) staining are colocalized on a pial vessel. Neuronal cell bodies on the pial vessel are AQP1-immunolabeled (arrow heads) and their NF-positive axons are surrounded by AQP1 immunolabeling (double lined arrows). $\mathrm{Bar}=25 \mu \mathrm{m}$. For interpretation of the references to color in this figure legend, the reader is referred to the Web version of this article. pression in pathological conditions. Human brain tissues were obtained after surgery (Badaut et al., 2003; Fatemi et al., 2008a; Saadoun et al., 2002a,b; Satoh et al., 2007; Suzuki et al., 2006) or autopsy (Satoh et al., 2007). To date, little is known about the distribution and expression of AQPs in normal primate brains. Here we report for the first time, the expression and cell localization of the three most studied AQPs in the rodent brain, AQP1, 4 and 9, in the Macaca fascicularis brain. We show that while the major rodent expression pattern is conserved for AQP4, there are some differences between rodents and non-human primates for AQP1 and 9. This knowledge is highly relevant for future development and testing of new drugs aimed to directly modify the permeability properties of AQPs to prevent edema formation in brain diseases.

\section{AQP4 distribution}

Similarly to previous description in rodent brains, AQP4 protein was found in perivascular astrocytic endfeet, and in contrast to AQP1, mainly in grey matter, but also in the glia limitans and pia mater. As previously described (Badaut et al., 2000b), we also noted high AQP4 expression in the 

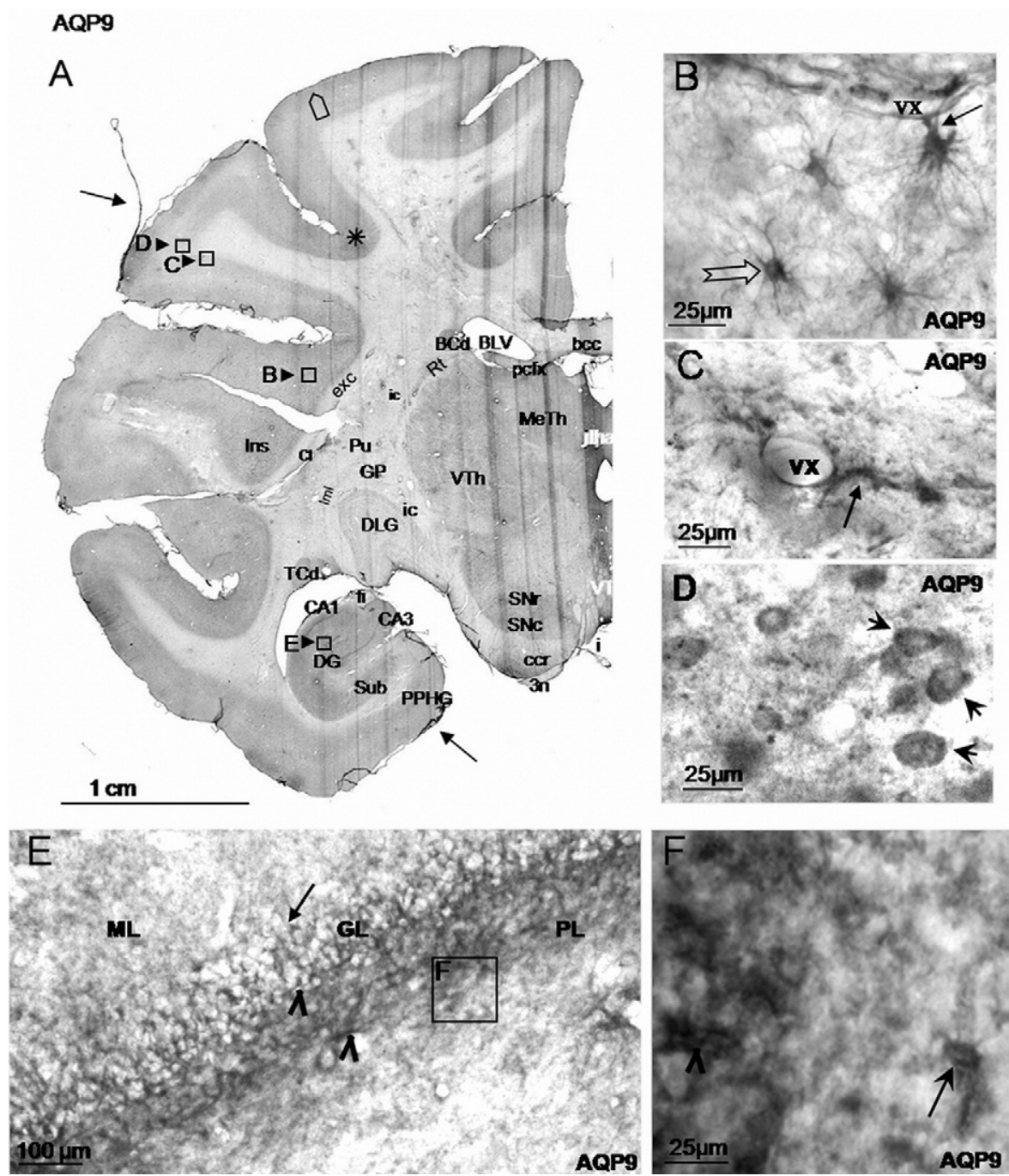

Fig. 7. AQP9 immunoreactivity in a coronal Macaca fascicularis brain section at $-6 \mathrm{~mm}$. (A) Staining is revealed in grey matter (asterix), in the Insula cortex (Ins), in the ventral (VTh), medial (MeTh) and reticular (Rt) thalamic nuclei, in the tail (TCd) and body (BCd) of the caudate nucleus, in the glia limitans (pentagonal arrows), in the pia mater (single lined arrows), in the substantia nigra compacta (SNc) and reticulata (SNr), in Cornu Ammonis fields CA1, CA2, CA3, in the dentate gyrus (DG) and in the subiculum (Sub) of the hippocampus. Compared to AQP1 labeling (Fig. 4) where the staining is mostly found in white matter and around the basal ganglia, AQP9 labeling is observed in the cortex and the basal ganglia system (except for the putamen and globus pallidus). The indicated squares are shown in higher magnification in the corresponding figures. 3n, oculomotor nerve; 3V, third ventricle; bcc, body of corpus callosum; BLV, body of lateral ventricle; ccr, cerebral crus; Cl, claustrum; DLG, dorsal nucleus of lateral geniculate body; ec, external capsule; exc, extreme capsule; GP, globus pallidus; Ins, Insula; ipf, interpeduncular fossa; itha, interthalamic adhesion; Iml, lateral medullary lamina; MeTh, medial thalamic nuclear group; pcfx, posterior column of fornix; Pu, putamen; PPHG, posterior parahippocampal gyrus; Sub, subiculum; VTA, ventral tegmental area. (B-D) Magnification of the cortical region of the precentral gyrus (PrG) as indicated in Fig. 7A, reveals expression of AQP9 in perivascular astrocytic endfeet (Fig. 7B, C, arrows) and in astrocytic cell bodies (Fig. 7B, double lined arrow) as well as in oval shaped cell bodies of 10 to $15 \mu \mathrm{m}$ of diameter (Fig. 7D, arrow heads). (E, F) The hippocampus region reveals high AQP9-IR in the granule cell layer. Higher magnification allows identification of immunolabeled cell bodies (open arrow heads) and processes (arrows) in the granule cell layer of the dentate gyrus. In Fig. 7F, higher magnification shows the cell bodies in the granule cell layer of small cells (open arrow heads) and bigger cells (arrow), suggesting the presence of two populations of cells. $\operatorname{Bar}=1 \mathrm{~cm}$ in $(A)$; $B a r=100 \mu \mathrm{m}$ in $(E)$; $\operatorname{bar}=25 \mu \mathrm{m}$ in $(B-D, F)$.

granule cell layer of the DG (Fig. 2D), limited to astrocytic endfeet. Generally, AQP4 labeling in primate brains is limited to astrocytic perivascular endfeet. In accordance to numerous previous reports in rodent brains (Badaut et al., 2007; Tait et al., 2008), we suggest that this specific AQP4 localization, in close contact to the cerebral blood vessels, as well as at the interface of the cerebrospinal fluid (CSF) - brain barrier, shows an important role in brain water homeostasis and in the formation and reab- sorption of brain edema. Our results highlight that brain AQP4 distribution is highly conserved between species from chicken (Goren et al., 2006; Saito et al., 2005) to primates (Fig. 2); suggesting that perivascular AQP4 plays a key role in brain water homeostasis in all species. AQP4 was also observed in the granule cell layer in the dentate gyrus of the hippocampus where we also noted expression of AQP1 and AQP9. Their potential roles will be discussed below. 

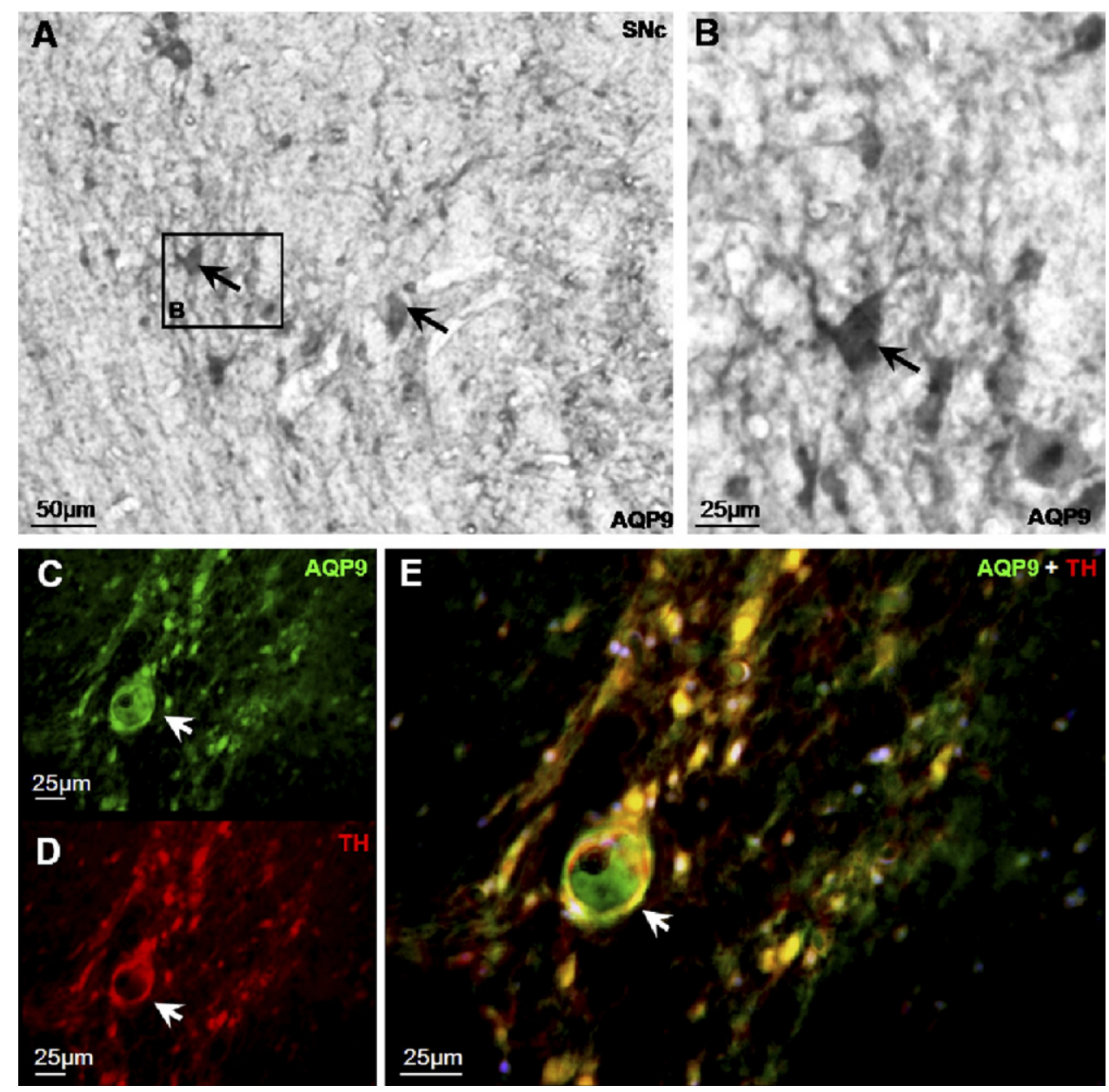

Fig. 8. (A, B) AQP9 immunolabeling in the substantia nigra compacta with positive cell bodies (arrows) in (A) and (B) (at higher magnification). Neuronal processes were also stained with AQP9 (B). (C-E) TH (red) and AQP9 (green)-double immunofluorescent staining of substantia nigra (Fig. 8E) reveals an AQP9-staining of cell bodies (Fig. 8C, arrow) with the same morphology as TH positive neurons (Fig. 8D, arrow), showing AQP9 expression in catecholaminergic neurons. We note both cytoplasmic and membrane expression of AQP9 (Fig. 8C, arrow), whereas TH is only expressed in the membrane of catecholaminergic neurons (Fig. 8D, arrow). Bar $=50 \mu \mathrm{m}$ in (A); Bar $=25 \mu \mathrm{m}$ in (B-E). For interpretation of the references to color in this figure legend, the reader is referred to the Web version of this article.

\section{AQP1 distribution and its potential roles}

As previously proposed for rodent brains (Nielsen et al., 1993; Oshio et al., 2005), AQP1 may contribute to cerebrospinal fluid formation in Macaca fascicularis brains. Whole brain analysis of AQP1 expression showed three additional locations in Macaca fascicularis: (1) in astrocytes, with a preference for white matter astrocytes (Fig. 4A); (2) in Schwann cells surrounding the oculomotor (Fig. $5 \mathrm{D}, \mathrm{E})$ and trigeminal (data not shown) cranial nerve fibers; and (3) in neurons on the surface of the pial blood vessels (Fig. 6B).

For the first time in Macaca fascicularis brain, we report AQP1 expression in astrocytic soma in white matter and the glia limitans as well as in their prolongations and vascular endfeet. In contrast to AQP4, only approximately one out of four GFAP-positive astrocytes were labeled by antiAQP1. AQP1 was preferentially expressed in astrocytes of the white matter, suggesting that AQP1 may be a marker of a sub-population of astrocytes. In grey matter, the most abundant type of astrocyte is the protoplasmic astrocyte. Human protoplasmic astrocytes have numerous processes and a 27-fold greater volume than protoplasmic astrocytes found in rodents (Oberheim et al., 2006). Fibrous astrocytes show less ramifications than protoplasmic astrocytes and have fewer GFAP-positive processes which, in contrast to protoplasmic processes, intermingle and do not respect a domain structure (Oberheim et al., 2006). In accordance with findings in human brain tissue (Satoh et al., 2007), our results in Macaca fascicularis brain suggest that AQP1 is a marker of fibrous astrocytes which seem to exhibit highly branched processes (Fig. $5 C)$. AQP1 may play a complementary role to AQP4 in astrocytes in the regulation of brain water homeostasis in primates.

Recently, expression of AQP1 in reactive astrocytes was shown in human brain pathologies, such as Alzheimer's disease, multiple sclerosis, ischemic brain lesions, brain edema and epilepsy (Badaut et al., 2003; Misawa et 
al., 2008; Satoh et al., 2007). Some studies have also shown an intracellular location for AQP1 in astrocytes (Dohke and Turner, 2002; Marinelli et al., 1997). In pathological conditions, AQP1 is proposed to play a role in brain water movement (Badaut et al., 2003; Satoh et al., 2007; Suzuki et al., 2006). However, AQP1 as well as AQP4 were recently shown to be involved in cell growth and migration (Saadoun et al., 2005a,b) and therefore, AQP1 in collaboration with AQP4 may play a role in glial scar formation by facilitating cell migration in pathological conditions. In normal primate brains, the presence of AQP1 in a single population of astrocytes suggests that this subtype may be dedicated to the rapid mobilization of cells after brain injury. This population of astrocytes with highly ramified processes is a population which detects modifications in the environment by rapid formation of filopodia. In pathological states, these AQP1 positive astrocytes could be more rapidly recruited to form reactive astrocytes. This hypothesis is coherent with observations of AQP1 in reactive astrocytes in several diseases (Badaut et al., 2003; Satoh et al., 2007; Suzuki et al., 2006).

Additional glial locations were also shown in peripheral nerves with GFAP-AQP1 co-expression in Schwann cells which envelop the oculomotor (Fig. 5D, E) and trigeminal (not shown) nerve fibers. The presence of AQP1 was previously reported in distant Schwann cells and also in human peripheral nerves (such as pancreatic and sciatic nerve bundles) (Gao et al., 2006; Nandasena et al., 2007). AQP4 expression was not observed in oculomotor or trigeminal nerve axons, suggesting that AQP1 is a marker of Schwann cells which myelinate nerve axons in the peripheral nervous system. As suggested above, AQP1 may be involved in water homeostasis in myelinated axons during electrical activity. However, more functional experiments will be necessary to determine the exact function of this channel in peripheral nerves.

AQP1 was also reported in primary sensory neurons in rodents and proposed to play a role in nociception, such as thermal and chemical pain reception (Oshio et al., 2006; Shields et al., 2007). In addition to these locations in rodent brains, we report for the first time that AQP1 is expressed in neuronal cell bodies and axons on pial vessels in monkeys (Figs. 5D, F and 6). Recent experiments in rodents showed that AQP1 is expressed in sensory neurons expressing substance $P$ (Shields et al., 2007). It is well known that the pial blood vessels are innervated by peptidergic (substance $P$ ) and nitrergic neurons coming from different ganglia, such as the trigeminal and sphenopalatin ganglia respectively (ladecola et al., 1994; Yamamoto et al., 1983). Taken together, we suggest that AQP1 protein is found in peptidergic and nitrergic neurons running on pial blood vessels. Recently, AQP1 was shown to facilitate the diffusion of nitric oxide (NO) and to contribute to vasorelaxation (Herrera and Garvin, 2007; Herrera et al., 2006), raising the hypothesis that neuronal AQP1 may contribute to NO diffusion from neurons to smooth muscle cells of pial blood vessels.

In summary, the biggest differences between rodents and non-human primates were observed for AQP1 distri- bution with new cell locations such as astrocytes, Schwann cells and pial blood vessel innervations.

\section{AQP9 distribution and its potential roles}

AQP9 is an aquaglyceroporin, which means it is also permeable to glycerol, urea and monocarboxylates, such as lactate. We have suggested a role for AQP9 in brain energy homeostasis (Badaut et al., 2007) and have described its expression in astrocytes and catecholaminergic neurons (Badaut and Regli, 2004). The level of expression in the brain as well as in the liver is regulated by plasma insulin concentrations, arguing for a role of AQP9 in energy metabolism (Badaut et al., 2008). However, immunocytochemical analyses have provided divergent results concerning the expression of AQP9 in brain. Its presence in mice brain was questioned (Rojek et al., 2007) but was also confirmed in rat and mouse tyrosine hydroxylasepositive neurons, in hilar and pyramidal cells of hippocampus as well as in numerous cell populations in the neocortex (Mylonakou et al., 2009). Mylonakou et al. (2009) studied several antibodies used in the various studies and concluded that the inconsistency between the immnocytochemical studies reflects differences between the specificity of antibodies used. They conclude that the AQP9 rabbit polyclonal antibody from Alpha Diagnostics produces the most reliable results (Mylonakou et al., 2009) and as with their studies, this study has been conducted with AQP9 rabbit polyclonal antibody from Alpha Diagnostics.

We demonstrate the presence of AQP9 mRNA in primate slices adjacent to those used in immunohistochemistry. The results are in agreement with previous studies in rodent brains (Badaut et al., 2004; Mylonakou et al., 2009), where we observe AQP9 protein in astrocyte cell bodies, processes and endfeet in grey matter (Fig. 7C, D) and in catecholaminergic neurons (Fig. 8). Here, we provide arguments for the expression of AQP9 in the non-human primate brain. However, the exact role of this aquaglyceroporin is still hypothetical and needs further investigation.

The presence of AQP9 was also observed in a second population of neurons in monkey brains with an oval shape in layer IV of the precentral gyrus (Fig. 7A) and the DG area of the hippocampus. This distribution is in accordance with the pattern of AQP9 mRNA distribution in mouse brains (Mylonakou et al., 2009). The functional role of AQP9 in these cells needs to be studied further.

Interestingly, the pattern of AQP9, AQP4 and AQP1 staining in the $D G$ is similar to the staining observed in the sub-ventricular zone in rodent brains (Cavazzin et al., 2006). The latter location is known to be a niche for adult progenitor cells (Cavazzin et al., 2006) as well as the DG of the hippocampus (Gage, 1998; Palmer et al., 1997; Song et al., 2002). The presence of AQP9, AQP4 and AQP1 in these regions suggests expression in progenitor cells. In agreement, previous studies have reported the presence of AQP9 and AQP4 in murine neural stem cells (NSC) (Cavazzin et al., 2006; La Porta et al., 2006). High expression of these AQPs (AQP1, AQP4, AQP9) in neural cell progenitor niches may be related to the high prolifer- 
ation rate in this brain area requiring fine tuning of both water and energy homeostasis.

\section{CONCLUSION}

In conclusion, the present study is the first to demonstrate the presence of the three major AQPs in Macaca fascicularis brains. AQP4 is the major aquaporin, expressed exclusively in astrocytes with a preferential location in perivascular astrocyte endfeet. We show an interesting distribution of AQP1 in a sub-population of astrocytes. Its role in these cells is not yet determined and needs further investigation. Our work suggests that results obtained in previous studies in pathological rodent models such as brain ischemia with AQPs cannot automatically be transposed to human clinical studies. We suggest that AQP4 is not involved alone in brain water homeostasis, but that AQP1 may also be part of the regulation process. AQP9 mRNA and proteins are present in primate brain, with additional locations in the cortex and hippocampus. Further investigation in brains with models of non-human primate pathologies will be extremely useful to determine possible roles of each AQP in edema and other pathophysiological mechanisms.

Acknowledgments-The authors are grateful to Prof E. Rouiller (University of Fribourg, Switzerland) for providing us the access to the monkey brain slices. The authors thank Ms Hamou, Ms Pythoud and Ms Ternon for their help in running some of the immunohistochemical experiments and DrM. Price for her critical reading of the manuscript. Supported by the Swiss Science Foundation (FN 3100AO-108001, and 31003A-122166), Swissheart Foundation, Novartis Research Foundation.

\section{REFERENCES}

Amiry-Moghaddam M, Lindland H, Zelenin S, Roberg BA, Gundersen BB, Petersen P, Rinvik E, Torgner IA, Ottersen OP (2005) Brain mitochondria contain aquaporin water channels: evidence for the expression of a short AQP9 isoform in the inner mitochondrial membrane. FASEB J 19(11):1459-1467.

Amiry-Moghaddam M, Ottersen OP (2003) The molecular basis of water transport in the brain. Nat Rev Neurosci 4(12):991-1001.

Badaut J, Brunet JF, Grollimund L, Hamou MF, Magistretti PJ, Villemure JG, Regli L (2003) Aquaporin 1 and 4 expression after human acute subarachnoid hemorrhage. Acta Neurochir Suppl 86:495498.

Badaut J, Brunet JF, Petit JM, Guerin CF, Magistretti PJ, Regli L (2008) Induction of brain aquaporin 9 (AQP9) in catecholaminergic neurons in diabetic rats. Brain Res 1188:17-24.

Badaut J, Brunet JF, Regli L (2007) Aquaporins in the brain: from aqueduct to "multi-duct." Metab Brain Dis 22(3-4):251-263.

Badaut J, Hirt L, Granziera C, Bogousslavsky J, Magistretti PJ, Regli L (2001) Astrocyte-specific expression of aquaporin-9 in mouse brain is increased after transient focal cerebral ischemia. J Cereb Blood Flow Metab 21(5):477-482.

Badaut J, Lasbennes F, Magistretti PJ, Regli L (2002) Aquaporins in brain: distribution, physiology, and pathophysiology. J Cereb Blood Flow Metab 22(4):367-378.

Badaut J, Nehlig A, Verbavatz J, Stoeckel M, Freund-Mercier MJ, Lasbennes F (2000a) Hypervascularization in the magnocellular nuclei of the rat hypothalamus: relationship with the distribution of aquaporin-4 and markers of energy metabolism. J Neuroendocrinol 12(10):960-969.
Badaut J, Petit JM, Brunet JF, Magistretti PJ, Charriaut-Marlangue C, Regli $L$ (2004) Distribution of Aquaporin 9 in the adult rat brain: preferential expression in catecholaminergic neurons and in glial cells. Neuroscience 128(1):27-38.

Badaut J, Regli L (2004) Distribution and possible roles of aquaporin 9 in the brain. Neuroscience 129(4):969-979.

Badaut J, Verbavatz JM, Freund-Mercier MJ, Lasbennes F (2000b) Presence of aquaporin-4 and muscarinic receptors in astrocytes and ependymal cells in rat brain: a clue to a common function? Neurosci Lett 292(2):75-78.

Cavazzin C, Ferrari D, Facchetti F, Russignan A, Vescovi AL, La Porta CA, Gritti A (2006) Unique expression and localization of aquaporin-4 and aquaporin-9 in murine and human neural stem cells and in their glial progeny. Glia 53(2):167-181.

de Castro Ribeiro M, Hirt L, Bogousslavsky J, Regli L, Badaut J (2006) Time course of aquaporin expression after transient focal cerebral ischemia in mice. J Neurosci Res 83(7):1231-1240.

Dohke Y, Turner RJ (2002) Evidence that the transmembrane biogenesis of aquaporin 1 is cotranslational in intact mammalian cells. J Biol Chem 277(17):15215-15219.

Dolman D, Drndarski S, Abbott NJ, Rattray M (2005) Induction of aquaporin 1 but not aquaporin 4 messenger RNA in rat primary brain microvessel endothelial cells in culture. J Neurochem 93(4): 825-833.

Elkjaer M, Vajda Z, Nejsum LN, Kwon T, Jensen UB, AmiryMoghaddam M, Frokiaer J, Nielsen S (2000) Immunolocalization of AQP9 in liver, epididymis, testis, spleen, and brain. Biochem Biophys Res Commun 276(3):1118-1128.

Fatemi SH, Folsom TD, Reutiman TJ, Lee S (2008a) Expression of astrocytic markers aquaporin 4 and connexin 43 is altered in brains of subjects with autism. Synapse 62(7):501-507.

Fatemi SH, Folsom TD, Reutiman TJ, Sidwell RW (2008b) Viral regulation of aquaporin 4, connexin 43, microcephalin and nucleolin. Schizophr Res 98(1-3):163-177.

Fatemi SH, Pearce DA, Brooks Al, Sidwell RW (2005) Prenatal viral infection in mouse causes differential expression of genes in brains of mouse progeny: a potential animal model for schizophrenia and autism. Synapse 57(2):91-99.

Gage FH (1998) Stem cells of the central nervous system. Curr Opin Neurobiol 8(5):671-676.

Gao H, He C, Fang X, Hou X, Feng X, Yang H, Zhao X, Ma T (2006) Localization of aquaporin-1 water channel in glial cells of the human peripheral nervous system. Glia 53(7):783-787.

Gomori E, Pal J, Abraham H, Vajda Z, Sulyok E, Seress L, Doczi T (2006) Fetal development of membrane water channel proteins aquaporin-1 and aquaporin-4 in the human brain. Int J Dev Neurosci 24(5):295-305.

Goren O, Adorjan I, Kalman M (2006) Heterogeneous occurrence of aquaporin-4 in the ependyma and in the circumventricular organs in rat and chicken. Anat Embryol (Berl) 211(2):155-172.

Herrera M, Garvin JL (2007) Novel role of AQP-1 in NO-dependent vasorelaxation. Am J Physiol Renal Physiol 292(5):F1443-F1451.

Herrera M, Hong NJ, Garvin JL (2006) Aquaporin-1 transports NO across cell membranes. Hypertension 48(1):157-164.

ladecola C, Pelligrino DA, Moskowitz MA, Lassen NA (1994) Nitric oxide synthase inhibition and cerebrovascular regulation. $\mathrm{J}$ Cereb Blood Flow Metab 14(2):175-192.

Iwasaki $\mathrm{Y}$, Mimuro M, Yoshida M, Hashizume $\mathrm{Y}$, Ito M, Kitamoto T, Wakayama Y, Sobue G (2007) Enhanced Aquaporin-4 immunoreactivity in sporadic Creutzfeldt-Jakob disease. Neuropathology 27(4):314-323.

Jessen KR, Mirsky R (1984) Nonmyelin-forming Schwann cells coexpress surface proteins and intermediate filaments not found in myelin-forming cells: a study of Ran-2, A5E3 antigen and glial fibrillary acidic protein. J Neurocytol 13(6):923-934.

Jessen KR, Morgan L, Stewart HJ, Mirsky R (1990) Three markers of adult non-myelin-forming Schwann cells, 217c(Ran-1), A5E3 and 
GFAP: development and regulation by neuron-Schwann cell interactions. Development 109(1):91-103.

Jung JS, Bhat RV, Preston GM, Guggino WB, Baraban JM, Agre P (1994) Molecular characterization of an aquaporin cDNA from brain: candidate osmoreceptor and regulator of water balance. Proc Natl Acad Sci U S A 91(26):13052-13056.

La Porta CA, Gena P, Gritti A, Fascio U, Svelto M, Calamita G (2006) Adult murine CNS stem cells express aquaporin channels. Biol Cell 98(2):89-94.

Lennon VA, Kryzer TJ, Pittock SJ, Verkman AS, Hinson SR (2005) IgG marker of optic-spinal multiple sclerosis binds to the aquaporin-4 water channel. J Exp Med 202(4):473-477.

Manley GT, Fujimura M, Ma T, Noshita N, Filiz F, Bollen AW, Chan P, Verkman AS (2000) Aquaporin-4 deletion in mice reduces brain edema after acute water intoxication and ischemic stroke. Nat Med 6(2):159-163.

Marinelli RA, Pham L, Agre P, LaRusso NF (1997) Secretin promotes osmotic water transport in rat cholangiocytes by increasing aquaporin-1 water channels in plasma membrane. Evidence for a secretin-induced vesicular translocation of aquaporin-1. J Biol Chem 272(20):12984-12988.

Misawa T, Arima K, Mizusawa H, Satoh J (2008) Close association of water channel AQP1 with amyloid-beta deposition in Alzheimer disease brains. Acta Neuropathol 116(3):247-260.

Mylonakou MN, Petersen PH, Rinvik E, Rojek A, Valdimarsdottir E, Zelenin S, Zeuthen T, Nielsen S, Ottersen OP, Amiry-Moghaddam M (2009) Analysis of mice with targeted deletion of AQP9 gene provides conclusive evidence for expression of AQP9 in neurons. J Neurosci Res 87:1310-1322.

Nandasena BG, Suzuki A, Aita M, Kawano Y, Nozawa-Inoue K, Maeda T (2007) Immunolocalization of aquaporin-1 in the mechanoreceptive Ruffini endings in the periodontal ligament. Brain Res 1157:32-40.

Nesic O, Lee J, Unabia GC, Johnson K, Ye Z, Vergara L, Hulsebosch CE, Perez-Polo JR (2008) Aquaporin 1-a novel player in spinal cord injury. J Neurochem 105(3):628-640.

Nielsen S, Nagelhus EA, Amiry-Moghaddam M, Bourque C, Agre P, Ottersen OP (1997) Specialized membrane domains for water transport in glial cells: high-resolution immunogold cytochemistry of aquaporin-4 in rat brain. J Neurosci 17(1):171-180.

Nielsen S, Smith BL, Christensen El, Agre P (1993) Distribution of the aquaporin CHIP in secretory and resorptive epithelia and capillary endothelia. Proc Natl Acad Sci U S A 90(15):7275-7279.

Oberheim NA, Wang X, Goldman S, Nedergaard M (2006) Astrocytic complexity distinguishes the human brain. Trends Neurosci 29 (10):547-553.

Oshio K, Watanabe H, Song Y, Verkman AS, Manley GT (2005) Reduced cerebrospinal fluid production and intracranial pressure in mice lacking choroid plexus water channel Aquaporin-1. FASEB J 19:76-78.

Oshio K, Watanabe H, Yan D, Verkman AS, Manley GT (2006) Impaired pain sensation in mice lacking Aquaporin-1 water channels. Biochem Biophys Res Commun 341(4):1022-1028.

Palmer TD, Takahashi J, Gage FH (1997) The adult rat hippocampus contains primordial neural stem cells. Mol Cell Neurosci 8(6):389404.

Papadopoulos MC, Manley GT, Krishna S, Verkman AS (2004) Aquaporin-4 facilitates reabsorption of excess fluid in vasogenic brain edema. FASEB J 18(11):1291-1293.

Rodriguez A, Perez-Gracia E, Espinosa JC, Pumarola M, Torres JM, Ferrer I (2006) Increased expression of water channel aquaporin 1 and aquaporin 4 in Creutzfeldt-Jakob disease and in bovine spongiform encephalopathy-infected bovine-PrP transgenic mice. Acta Neuropathol 112(5):573-585.

Rojek AM, Skowronski MT, Fuchtbauer EM, Fuchtbauer AC, Fenton RA, Agre P, Frokiaer J, Nielsen S (2007) Defective glycerol metabolism in aquaporin 9 (AQP9) knockout mice. Proc Natl Acad Sci U S A 104(9):3609-3614.

Saadoun S, Papadopoulos MC, Davies DC, Bell BA, Krishna S (2002a) Increased aquaporin 1 water channel expression in human brain tumours. Br J Cancer 87(6):621-623.

Saadoun S, Papadopoulos MC, Davies DC, Krishna S, Bell BA (2002b) Aquaporin-4 expression is increased in oedematous human brain tumours. J Neurol Neurosurg Psychiatry 72(2):262-265.

Saadoun S, Papadopoulos MC, Hara-Chikuma M, Verkman AS (2005a) Impairment of angiogenesis and cell migration by targeted aquaporin-1 gene disruption. Nature 434(7034):786-792.

Saadoun S, Papadopoulos MC, Watanabe H, Yan D, Manley GT, Verkman AS (2005b) Involvement of aquaporin-4 in astroglial cell migration and glial scar formation. J Cell Sci 118(Pt 24):56915698.

Saito N, Ikegami H, Shimada K (2005) Effect of water deprivation on aquaporin 4 (AQP4) mRNA expression in chickens (Gallus domesticus). Brain Res Mol Brain Res 141(2):193-197.

Satoh J, Tabunoki H, Yamamura T, Arima K, Konno H (2007) Human astrocytes express aquaporin-1 and aquaporin-4 in vitro and in vivo. Neuropathology 27(3):245-256.

Shields SD, Mazario J, Skinner K, Basbaum Al (2007) Anatomical and functional analysis of aquaporin 1, a water channel in primary afferent neurons. Pain 131(1-2):8-20.

Song HJ, Stevens CF, Gage FH (2002) Neural stem cells from adult hippocampus develop essential properties of functional CNS neurons. Nat Neurosci 5(5):438-445.

St Hillaire C, Vargas D, Pardo CA, Gincel D, Mann J, Rothstein JD, McArthur JC, Conant K (2005) Aquaporin 4 is increased in association with human immunodeficiency virus dementia: implications for disease pathogenesis. J Neurovirol 11(6):535-543.

Suzuki R, Okuda M, Asai J, Nagashima G, Itokawa H, Matsunaga A, Fujimoto T, Suzuki T (2006) Astrocytes co-express aquaporin-1, -4 , and vascular endothelial growth factor in brain edema tissue associated with brain contusion. Acta Neurochir Suppl 96:398401.

Tait MJ, Saadoun S, Bell BA, Papadopoulos MC (2008) Water movements in the brain: role of aquaporins. Trends Neurosci 31(1): 37-43.

Tsukaguchi H, Shayakul C, Berger UV, Mackenzie B, Devidas S, Guggino WB, van Hoek AN, Hediger MA (1998) Molecular characterization of a broad selectivity neutral solute channel. J Biol Chem 273(38):24737-24743.

Verkman AS, Binder DK, Bloch O, Auguste K, Papadopoulos MC. (2006) Three distinct roles of aquaporin-4 in brain function revealed by knockout mice. Biochim Biophys Acta 1758:1085-1093.

Weinshenker BG, Wingerchuk DM, Pittock SJ, Lucchinetti CF, Lennon VA (2006) NMO-IgG: a specific biomarker for neuromyelitis optica. Dis Markers 22(4):197-206.

Yamamoto K, Matsuyama T, Shiosaka S, Inagaki S, Senba E, Shimizu Y, Ishimoto I, Hayakawa T, Matsumoto M, Tohyama M (1983) Overall distribution of substance P-containing nerves in the wall of the cerebral arteries of the guinea pig and its origins. J Comp Neurol 215(4):421-426. 